\title{
CIBERSEGURANÇA: IDENTIFICAÇÃO DE KEYSTROKE POR DISPOSITIVO RUBBER DUCKY ${ }^{1}$ \\ CYBERSECURITY: KEYSTROKE IDENTIFICATION BY RUBBER DUCKY DEVICE
}

\author{
Anderson Vagner Kobs², Sylvio André Garcia Vieira ${ }^{3}$
}

\section{RESUMO}

Com a digitalização de dados pessoais e corporativos a segurança da informação é pauta cada vez mais presente nas organizações. De encontro a esta abordagem este projeto traz uma solução para a identificação de dispositivo forense, denominado Rubber Ducky. Conhecido por executar scripts automatizados no momento da sua conexão em portas USB, esta aplicação, baseada em linguagem, impede a conclusão destes scripts, protegendo o usuário de uma eventual violação de confidencialidade documental digital.

Palavras-chave: Tecnologia, Segurança, Prevenção.

\section{ABSTRACT}

With the personal and corporate data digitalization, information security is an increasingly common issue in organizations. Against this approach, this project brings a solution for forensic device identification, called Rubber Ducky. Known for executing automated scripts when connecting to USB ports, this application, based on language, will prevent the completion of these scripts, protecting the user from an eventual breach of digital document confidentiality.

Keywords: Technology, Security, Prevention.

1 Trabalho de Final de Graduação II - TFG II.

2 Acadêmico do Curso de Sistemas de Informação - Universidade Franciscana. E-mail: inf.avagner@gmail.com. 3 Orientador - Professor do curso de Sistemas de Informação - Universidade Franciscana. E-mail: sylvio@ufn.edu.br. 


\section{INTRODUÇÃO}

A presença da tecnologia em tarefas rotineiras está cada vez mais em evidência. Equipamentos, antes compostos por uma série de componentes interligados, estão se tornando portáteis e de fácil transporte para qualquer lugar, carregando em seu interior um grande volume de informações. Muitas vezes estas informações possuem em seu teor uma confidencialidade muito elevada, pois podem representar o futuro de uma inovação, grandes prejuízos materiais, ou ser motivação de estelionatos, por exemplo.

A necessidade de uma educação adequada para um uso consciente dos sistemas digitais é de extrema importância. Tal fato evidencia a importância da correta instrução do fator humano sobre segurança digital, pois a decisão de ação no momento mais oportuno, da ferramenta mais adequada à situação, e da crucialidade da segurança dos dados ainda são de decisão do gerente dos sistemas (CARNEIRO, 2013). Falhas minuciosas em algumas destas etapas podem representar o momento perfeito para captura de dados por pessoas não autorizadas, interessadas em obter o máximo de vantagem enquanto detentoras destas informações.

A sofisticação da tecnologia apresenta uma certa dualidade. Enquanto o tráfego de informações consiste no envio e recebimento de dados criptografados, grandes empresas do ramo tecnológico empregam expressivo esforço em técnicas que mantenham seguras todo o volume informacional. Esta segurança deve ser pertinente tanto no momento em que transitam de um lado para outro, bem como o seu acesso restrito somente a pessoas autorizadas depois de armazenadas.

Mesmo com toda esta preocupação, com igual intensidade, pessoas mal-intencionadas buscam aprimorar suas estratégias na prática de apropriação indevida de informação alheia com alguma vantagem que esta pode proporcionar. Os ataques cibernéticos podem ser disparados com as mais diversas finalidades, como captura de senhas, fraudes bancárias, obtenção de arquivos pessoais, entre outros.

Considerando esta contínua sofisticação em invasão de dispositivos digitais e a conseqüente oferta de ferramentas e treinamentos tem-se como objetivo geral deste trabalho apresentar possíveis conseqüências desencadeadas pelo uso de equipamentos e sistemas hacking, como o dispositivo Rubber Ducky e ao mesmo tempo promover uma forma de proteção contra possíveis ações de obtenção de dados ilícitos a partir de um sistema computacional.

\section{SEGURANÇA DA INFORMAÇÃO}

Inevitavelmente todo ambiente institucional necessita estar interligado ao mundo externo para expandir as suas atividades, por meio de alguma forma de comunicação. Essa informação que circula entre as partes interessadas possivelmente estará sujeita a alguma interceptação. Assim sua gestão deve estar promovida de maneira estratégica para garantir o funcionamento e crescimento de toda a composição organizacional. 
No que ser refere a sua segurança, é imprescindível a identificação de vulnerabilidades que ofereçam algum tipo de risco a qualquer ativo de informação dentro de uma corporação (KONZEN, 2013). $\mathrm{O}$ armazenamento ainda ocorre das mais variadas formas ou meios, em dispositivos locais ou em rede, ou ainda em meio digital ou impresso. Apesar de ser armazenada em equipamentos tecnológicos, o autor enfatiza ainda que a segurança da informação depende ao mesmo tempo de uma gestão humana, capaz de manter o ambiente controlado, com políticas de restrição adequada. Deste modo será assegurado que o usuário destinado ao controle de determinada informação conduza o sistema de maneira eficiente e segura. Contemplando este conceito de gestão da Segurança da Informação, a seguridade da informação estará efetiva quando executado o sistema de gestão apresentado na Figura 1 (SILVA, 2009).

Figura 1 - Os três principais recursos na gestão da informação

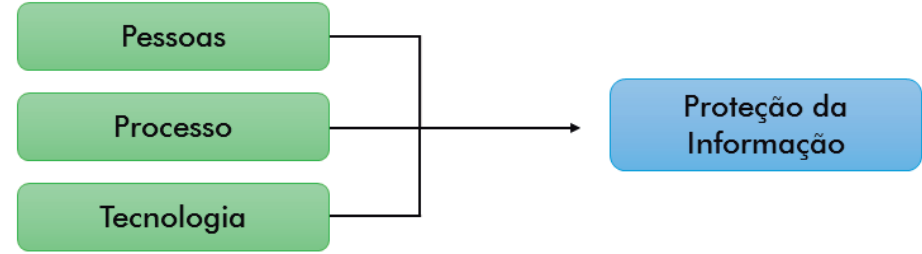

Fonte: Adaptado de SILVA, 2019.

Considerando a abordagem do conceito de Segurança da Informação sob as suas mais variadas perspectivas, entende-se que o armazenamento da informação é destinado a alguma finalidade em algum momento durante sua vida útil. Para garantir o cumprimento destes requisitos, a autora enfatiza que a segurança estará assegurada pela organização de três pilares: Disponibilidade, Integridade e Confidencialidade, destacados na Figura 2.

Figura 2 - Tripé da Segurança da Informação

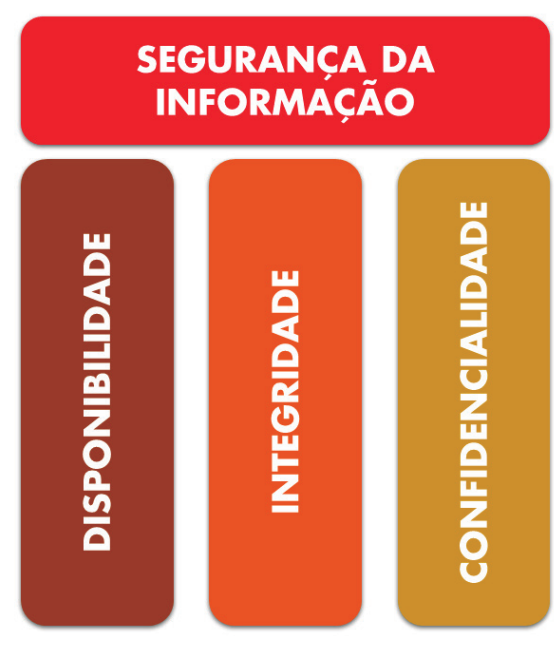

Fonte: Adaptado de SILVA, 2019.

Seguindo estes conceitos a qualidade da informação estará assegurada, garantindo-se sigilo, acesso e solidez a quem interessar, no momento oportuno. Qualquer violação de um destes itens pode 
resultar em perdas variadas a respeito das informações solicitadas, como por exemplo, vazamento de dados de caráter sigiloso da própria instituição proprietária ou de seus clientes.

Quanto a classificação das ameaças referente a Segurança da Informação, há alguns conceitos importantes (MARCIANO, 2006):

- Ameaças: segundo o autor, embasa-se em eventos indesejáveis aos equipamentos de informação cujas finalidades consistem em remover, danificar ou destruir algum recurso ou informação;

- Vulnerabilidades: quando um sistema de informação é elaborado podem ocorrer pontos falhos com passividade de exploração por alguma ameaça. Pode ser em pontos físicos no momento da sua instalação, ou ainda algum software com comunicação aberta configurado por um usuário;

- Ataques: representam alguma forma de ação muitas vezes meticulosamente planejada normalmente originadas por atuação humana. As tentativas de acesso indevido geralmente consistem em apropriação de informações indevidas, mas nem sempre completam o seu objetivo.

\section{PENTEST (TESTE DE PENETRAÇÃO)}

O risco de ataques a sistemas empresariais cresce a nível mundial na medida que toda a informação se transforma para a plataforma digital. A preocupação frente a estas ameaças tem motivado analistas e gerentes de segurança da informação a buscar soluções de precaução antes que um hacker promova algum tipo de dano por uma invasão.

A busca por soluções que promovam uma segurança eficiente frente a estas ameaças motivou especialistas da área de Segurança da Informação na promoção de técnicas cujo objetivo esteja sempre um passo à frente da ação dos hackers. Esta rotina de testes prévios na identificação de vulnerabilidades é conhecida atualmente como Teste de Penetração, ou da abreviação em inglês "Pentest".

Um Teste de Penetração consiste em um padrão de técnicas de alto nível de segurança, com finalidade de encontrar potenciais vulnerabilidades em uma infraestrutura de tecnologia, seja um sistema, servidor ou uma estrutura de rede em geral (ROCHA et al., 2016). Este tipo de ação também pode ser pontual, direcionando-a a apenas itens cruciais, como por exemplo dados corporativos com registros estritamente sigilosos. Mais do que uma metodologia, a eficiência dos testes fornece um relatório com todas as informações minimizando o impacto de potenciais ataques (PAVAN; GUARDIA, 2015). 
A sua classificação é baseada no tipo de complexidade e informação disponível no momento do procedimento. A quantidade de informações no momento do ataque pode fazer muita diferença (ROCHA et al., 2016). Podem ser:

- Blind: neste procedimento o auditor não tem nenhuma informação sobre o alvo, mas o alvo sabe que será atacado e os procedimentos que serão executados;

- Double Blind: neste processo ninguém tem informações, o auditor não conhece nada sobre o alvo, nem o alvo não sabe que será vítima de ataque, nem dos procedimentos que serão executados;

- Gray Box: o auditor tem um pouco de informações sobre o alvo, o alvo está notificado sobre o ataque no qual ele será vítima, e também sabe sobre os procedimentos que serão efetuados;

- Double Gray Box: o auditor tem algumas informações sobre o alvo, o alvo tem conhecimento sobre o ataque, mas não sabe sobre os procedimentos que serão executados;

- Tandem: o auditor tem informações detalhadas sobre sua vítima, o alvo sabe que será atacado e quais as tarefas que serão executadas;

- Reversal: o auditor tem detalhes sobre o alvo, mas ele não sabe sobre o ataque, nem dos procedimentos que serão executados.

\section{ETAPAS DE UM PENTEST}

Como qualquer planejamento de uma operação em um sistema de TI a ação de um pentest é composta por etapas, de modo que haja uma maior eficiência durante a sua execução. Dentre as referências consultadas há uma pequena discrepância na nomenclatura, mas os processos envolvidos são praticamente os mesmos.

As etapas a serem seguidas são (SANTOS, 2018):

- Preparação: refere-se a todos os procedimentos de comum acordo entre o pentester ${ }^{4} \mathrm{e}$ o cliente. Neste escopo serão definidos todos os processos esperados e seus possíveis resultados depois da execução. Dependendo do tipo de teste, conforme classificação apresentada anteriormente, serão levados em conta detalhes como a infraestrutura e os pontos onde deverá haver mais atenção.

- Coleta de informações: consiste na aquisição de informações sobre a situação do cliente. A obtenção destas informações direcionará o analista pelos meios a serem explorados.

\footnotetext{
4 Profissional com elevado conhecimento sobre técnicas e ferramentas utilizadas em identificação de falhas de segurança em sistemas de informação e infraestrutura de redes.
} 
- Modelagem das ameaças: baseia-se no desenvolvimento do plano de ataque. Dependendo do tipo de informação da organização, o plano de ação será direcionado aos elementos de crucial importância para sua existência.

- Análise de vulnerabilidades: nesta etapa virão à tona todas as deficiências em segurança do sistema. Poderão ser expostos todos os tipos de ações com possibilidade de interrupção dos processos críticos.

- Exploração das falhas: fase onde todas as vulnerabilidades encontradas durante o teste são acessadas.

- Pós-exploração de falhas: momento onde todas as vantagens que poderão ser obtidas pelo atacante são expostas.

- Geração de relatórios: depois de executados todos os procedimentos de invasão chega-se ao momento de recolher todas as informações úteis e essenciais para o cliente na melhoria de seus sistemas de segurança da informação.

Estas etapas conduzem a ação de análise de maneira clara e coerente tanto para o analista, como para o cliente, promovendo uma maior integração durante o processo.

\section{USB RUBBER DUCKY}

O padrão de conexão USB trouxe grandes benefícios no âmbito de conexões de periféricos a computadores. Antigamente essa conexão era tarefa de técnicos ou usuários com um significado nível de conhecimento em interconexões dispositivos.

Quando um dispositivo for conectado ao host (computador) ele faz uma série de solicitações ao dispositivo conectado, como informações básicas de tipo, tamanho, etc. (FILHO, 2018). Com o driver adequado instalado pelo host o dispositivo USB passa para o modo execução e assim o firmware passa a controlar a função básica do periférico. Esse firmware vem geralmente programado de fábrica, e no momento da sua aquisição presume-se que ele seja executado adequadamente conforme a sua finalidade.

Há ainda um outro problema, que se refere a possibilidade de reprogramação de firmware de alguns dispositivos. Isso se deve à falta de segurança de implementação para que se evite a reprogramação destas instruções. A possibilidade de programação de instruções para ser reconhecidas no momento da conexão via porta USB é o princípio de funcionamento do dispositivo Rubber Ducky.

Fisicamente trata-se de um dispositivo com uma conexão USB, que no momento do seu acoplamento a porta USB do host, poderá executar um payload como uma diversidade de finalidades, como por exemplo, abertura de um backdoor para exploração da infraestrutura, ou apenas recolher determinados arquivos de interesse. A linguagem de programação utilizada para criar o script é o Ducky Script. A Figura 3 apresenta em detalhes a sua composição de hardware. 
Figura 3 - Dispositivo Rubber Ducky utilizado nos testes, fabricante HAK5.

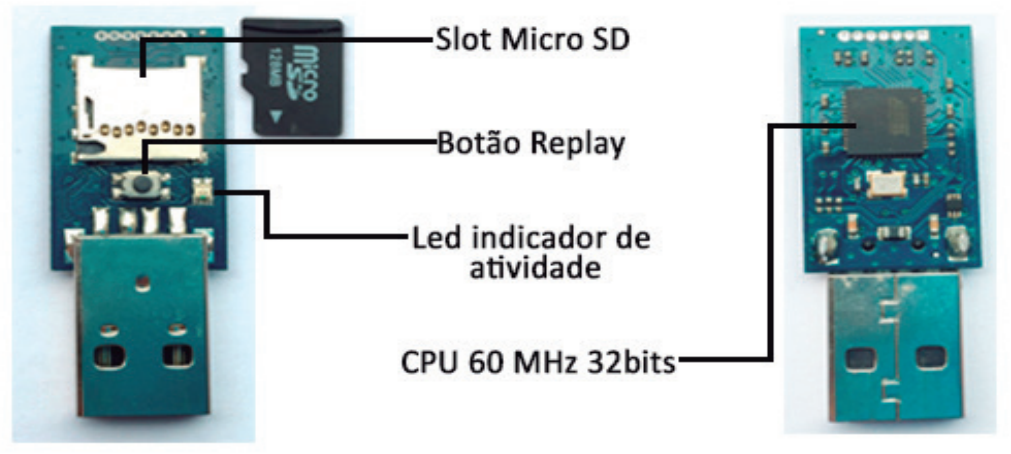

Fonte: Construção do autor.

Equipado com um micro controlador de $60 \mathrm{MHz}$ e uma arquitetura de 32 bits, pode executar scripts com uma grande variedade de finalidades.

\section{DUCK TOOLKIT}

O Duck Toolkit trata-se de uma série de ferramentas que permitem codificar e descodificar os arquivos responsáveis por realizar a execução dos comandos do dispositivo Rubber Ducky. Disponível em sua homepage, após inserido o script com as funcionalidades a serem desempenhadas, permite a geração do inject.bin. Na sequência da geração do arquivo, o mesmo deve ser baixado e salvo no microSD que é inserido na placa do Rubber Ducky. Se for necessário o script inserido também pode ser salvo ainda em formato de texto.

A ferramenta também possui alguns modelos pré-definidos, os quais podem servir de teste ou exemplo durante o desenvolvimento do script com os comandos. Em seu menu lateral a direita pode-se observar todos os comandos responsáveis pela codificação do arquivo inject.bin. O seu funcionamento é simples. Cada nova linha é responsável pela execução de um comando. Na sequência segue uma exemplificação de um código.

Figura 4 - Sintaxe básica para a criação de um arquivo inject.bin.

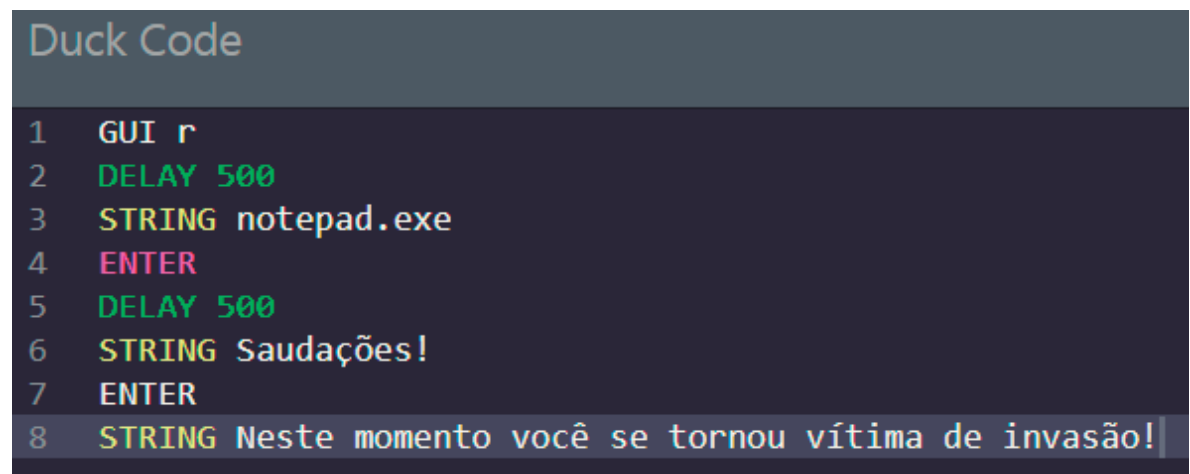

Fonte: Construção do autor. 


\section{LINGUAGEM DE PROGRAMAÇÃO PYTHON}

Em 2001 foi fundada a PSF (Software Foundation), a qual trata-se de uma organização sem fins lucrativos, coordenadora do uso da linguagem até hoje. Recebe apoio de grandes empresas do setor de tecnologia, como Google, Microsoft e a Globo.com.

Sobre a sua aplicação, trabalha de forma isolada, porém pode ser integrada a outras linguagens, como Java, C, JavaScript, entre outras (SILVA; SILVA, 2019).

De uma maneira generalista trata-se de uma linguagem de altíssimo nível, concretizando uma premissa de seu criador, o qual projetou-a para ser de fácil entendimento por programadores novos interessados em aprendê-la.

\section{PY2EXE}

O módulo Py2exe consiste em uma extensão para a linguagem que converte scripts (.py) em executáveis para o Sistema Operacional Microsoft Windows (PY2EXE, 2020). Após a conversão os executáveis gerados podem ser executados de forma independente, onde o interpretador não necessita estar instalado.

Para seu funcionamento correto, durante a sua instalação é necessário estar atento a características como a arquitetura do Sistema Operacional (32 ou 64 bits), bem como a versão da linguagem instalada.

\section{PYHOOK}

Também consiste em mais um módulo criado para a linguagem Python (PYHOOK, 2020). Esta extensão tem a finalidade de fornecer retorno e atividades executadas por teclado e mouse de computador. Registra as atividades onde o usuário executa tarefas como ação do mouse direito, esquerdo e tecla digitada. Associado a isso pode retornar informações como data e hora da ação.

\section{TRABALHOS CORRELATOS}

Neste item serão apresentados alguns trabalhos desenvolvidos dentro da temática de invasão, utilizando técnicas baseadas no dispositivo Rubber Ducky e as formas de exploração de vulnerabilidades na execução destes scripts automatizados. 
Neste trabalho de Cannols e Ghafarian (2017), os autores abordaram de maneira prática a utilização da ferramenta Rubber Ducky. Seu uso, em apoio de mais algumas técnicas de intrusão mostraram como alguns segundos de desatenção são suficientes para exploração de falhas. Neste caso aproveitaram a situação de falha apresentada pelo Sistema Operacional Windows por possibilitar a obtenção das informações referentes a "Id" e "Senha" dos usuários cadastrados (CANNOLS; GHAFARIAN, 2017).

Aliado ao uso do dispositivo Rubber Ducky, os testes no cenário obtiveram apoio de ferramentas como: Mimikatz, uma ferramenta de código aberto que permite a exploração do Windows LSASS (Local Security Authority Subsystem Service) através do seu módulo sekurlsa, o qual possui uma série de informações, entre os quais estão a "Id" e "Senha" de usuário. A sua execução permite coletar todas as informações desta “.dll”. Um detalhe muito importante refere-se a sua segurança, pois este aplicativo é detectado pelo antivírus.

De acordo com a descrição das ferramentas, e quanto ao roteiro deste trabalho, com o uso do DuckyScript foi programado o "inject.bin” adicionado ao dispositivo Rubber Ducky. Quando conectado em uma porta USB possibilitou a execução do PowerShell como administrador, e a partir dele uma série de outros comandos, como execução da ferramenta Mimikatz e envio deste log ao servidor de hospedagem definido pelos programadores.

A conclusão disso infere que, apesar deste teste tirar proveito de uma falha do Windows, vários outros riscos podem ser submetidos por um pequeno descuido, permitindo a ação de pessoas mal-intencionadas, bem como critérios e políticas de segurança no manuseio de dispositivos desconhecidos em ambientes com informações confidenciais, tanto pessoais como corporativos.

\section{WI-FI PASSWORD STEALING PROGRAM USING USB RUBBER DUCKY}

Neste trabalho também envolvendo o dispositivo Rubber Ducky, Harianto e Gunawan realizaram um teste de penetração com o objetivo de capturar a senha de WI-FI a qual o computador está conectado. Foram usadas algumas ferramentas de apoio, mas o Rubber Ducky foi usado como dispositivo desencadeante da ação (HARIANTO; GUNAWAN, 2018).

Segundo a Figura 5, o roteiro da pesquisa foi baseado em 3 passos principais.

Figura 5 - Fluxograma de passos para a captura de senhas WI-FI.

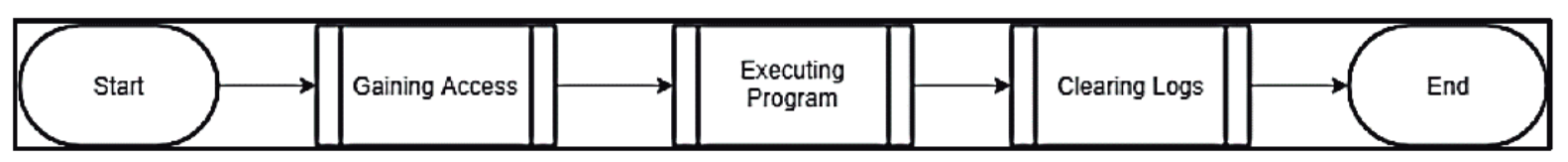

Fonte: Adaptado de HARIANTO; GUNAVAM, 2018. 
Com o acesso a máquina física todos os passos restantes são executados no código armazenado pelo inject.bin. Uma ação clássica deste tipo de invasão faz uso de transferência de arquivos online para servidores via FTP (File Transfer Protocol), exatamente conforme aplicado neste projeto.

Durante a sua ação foram realizadas basicamente as seguintes tarefas:

1. Abertura de prompt de comando como administrador;

2. Desativação de Firewall;

3. Extração das informações de senha com o comando "netsh wlan export profile key=clear" e exportação para arquivo XML;

4. Compressão para arquivo .ZIP;

5. Upload de arquivo via FTP;

6. Eliminação dos arquivos e diretórios criados; ativação de Firewall;

7. Fechamento de prompt de comando.

Durante a análise dos resultados deste trabalho foi ainda possível avaliar o nível de força utilizado pelos usuários em suas senhas. A aplicação dos testes foi realizada por uma totalidade de 35 vezes, onde os resultados obtiveram sucesso em 33 vezes. Em 2 execuções ocorreu falha na captura pelo motivo da senha conter caracteres além de números e letras alfabéticas. Com base nisso pode ser destacada a importância de uma senha com nível apropriado de segurança.

\section{METODOLOGIA}

Conforme apresentado anteriormente, o dispositivo Rubber Ducky consiste na execução de um payload automatizado, programado por uma linguagem própria. O disparo da sua execução se sucede depois da sua conexão a uma porta USB de uma máquina física. Primeiramente tornou-se necessário o estudo da linguagem responsável pelos seus comandos, o Ducky Script. A partir de sua sintaxe foram elaboradas as rotinas e comandos. Ao finalizar as instruções é criado o arquivo responsável pela ação no momento da sua conexão, o "inject.bin".

A implementação desta solução de defesa teve como princípio norteador a realização de teste originado a partir de um projeto open-source orientado e disponibilizado por Pedro Miguel Sosa. Pedro é estudante de segurança e criptografia em computação pela Universidade de Califórnia, em Santa Bárbara/EUA. De maneira bem didática Pedro instiga o leitor a tentar compreender como um dispositivo projetado para a computação forense como um Rubber Ducky pode ser identificado.

Considerando os princípios de funcionamento deste dispositivo, podemos nos indagar com o seguinte questionamento: como identificar uma invasão por Rubber Ducky se no momento da sua conexão a uma porta USB um computador reconhece-o como um dispositivo de entrada, como um teclado? Como impedir uma série de comandos automatizados por um script se nem o mais sofisticado antivírus não possui capacidade de identifica-lo? 
Com o objetivo de preencher esta lacuna de segurança, e para comprovação se de fato esta solução funcionaria, foi adquirido o dispositivo oficial da fabricante HAK5, uma das mais conceituadas empresas na fabricação deste tipo de hardware. Infelizmente em seu site oficial (shop.hak5.org) não há exportação direta para o Brasil, porém por intermédios foi realizada a sua aquisição.

Baseado em seu blog pessoal e GitHub, Pedro Sosa traz uma reflexão para identificar este tipo de invasão baseado em sua forma de funcionamento. Quando um Rubber Ducky é conectado fisicamente a um host começa a execução de script, o qual em algum momento certamente vai disparar um comando baseado em uma string de caracteres. Conhecendo alguns dos comandos básicos do Sistema Operacional pode-se caracterizar cada comando com uma média de 10 a 20 caracteres. Então neste ponto chega-se a um momento chave no desenvolvimento deste projeto: identificar se o comando é humanamente possível de ser digitado dentro de um instante de tempo.

A linguagem de programação Python possui módulos que fazem a captura de eventos de teclado e mouse. Sabendo-se da execução extremamente rápida de um script por um Rubber Ducky, este módulo terá a responsabilidade de armazenar um histórico com a entrada de caracteres. Se a velocidade de entrada for maior que o tempo estipulado, necessariamente haverá uma interrupção da entrada de caracteres, deixando a execução do script incompleta.

Para isso foram elaborados 3 arquivos e identificados como:

- duckhunt.conf: arquivo de configuração, o qual pode ser personalizado pelo usuário. Ao abri-lo com algum editor de texto haverá duas variáveis que podem ser editadas, estipulando a quantidade de caracteres monitorados e o espaço de tempo em milissegundos para a identificação;

- $\quad$ setup.py: arquivo necessário para a compilação de executável para o Sistema Operacional Windows pelo módulo Pyzexe;

- duckunt_Ajustável.py: este é o arquivo que contém a programação responsável pela identificação da invasão. As importações estão destacadas na Figura 6.

Figura 6 - Importação dos módulos Python, com destaque ao módulo PyHook.

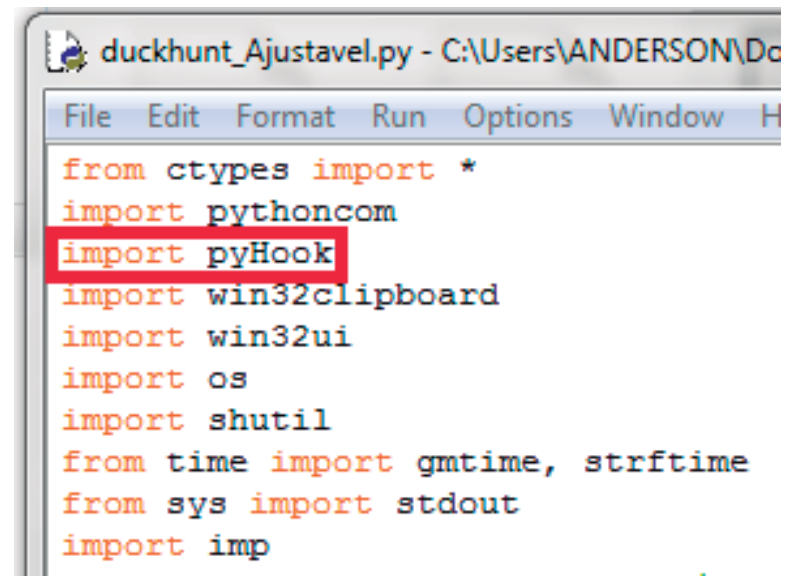

Fonte: Construção do autor. 
Depois da compilação do executável com o módulo Py2exe, são gerados dois diretórios: um denominado "build" e outro "dist". A pasta "dist" conterá o executável que deverá ser executado. Basta executá-lo e finalmente dentro dos processos do Windows, iniciará um processo com o mesmo nome. A partir deste momento o Sistema Operacional estará protegido.

Como o objetivo deste trabalho consiste em encontrar uma solução que de alguma maneira realize o bloqueio da ação deste tipo de dispositivo, o script criado não realizará tarefas muito complexas. A Figura 7 apresenta o seu script criado com a ferramenta on-line Duck Toolkit.

Figura 7 - Elaboração do Ducky Script, o qual dará origem ao arquivo inject.bin.

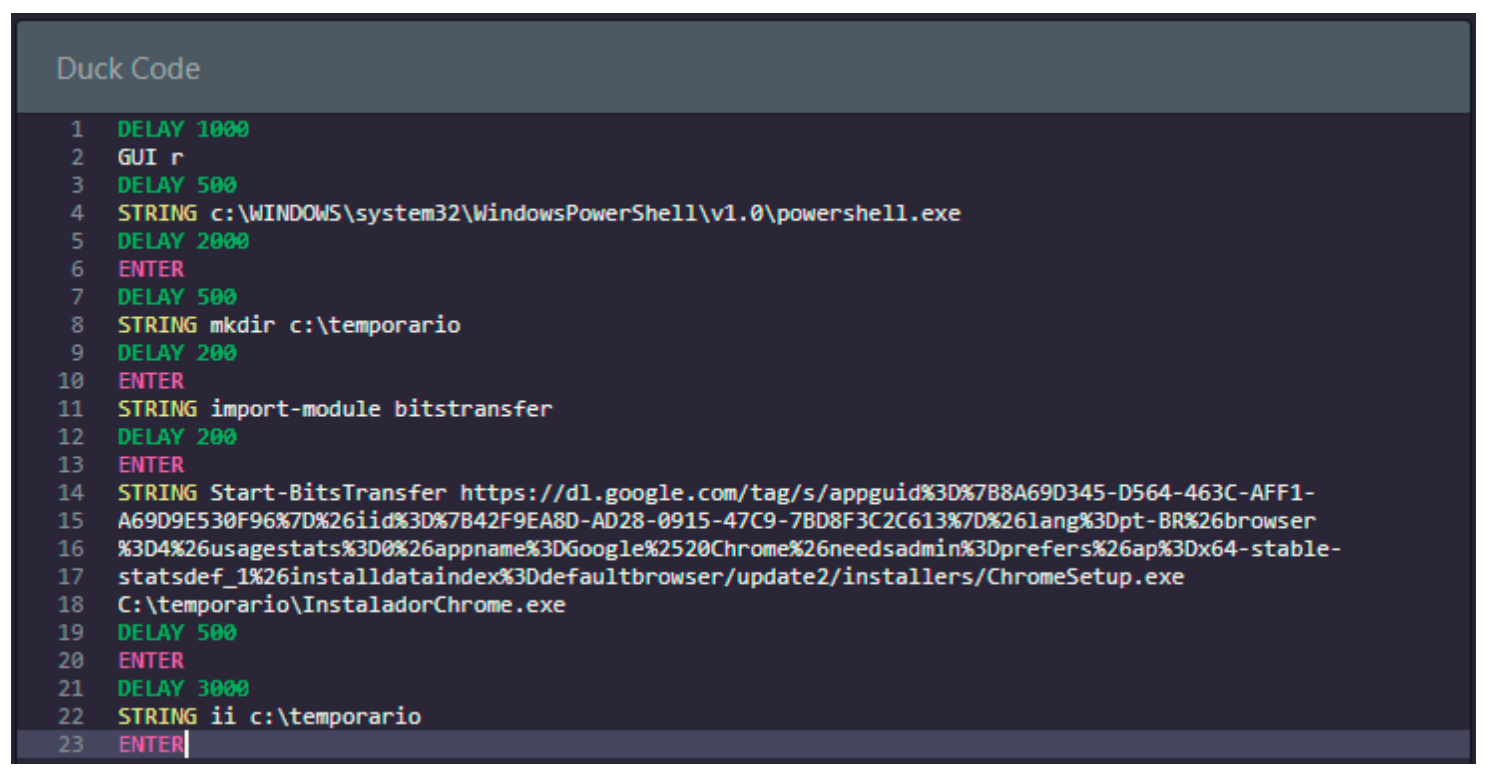

Fonte: Construção do autor.

Uma das tarefas mais comuns realizadas com este tipo de hardware consiste em transferência de aplicações a partir de servidores on-line via FTP e utilização de comandos robustos via PowerShell. Para demostrar de maneira didática como o processo de transferência de arquivos é simples, o script acima define a execução sequencial das seguintes tarefas:

1. Abertura da caixa de diálogo "Executar" no Windows;

2. Abertura do Windows PowerShell;

3. Criação da pasta "temporario" no disco local C:l, para onde o arquivo será baixado;

4. Criação de um serviço de transferência de arquivos;

5. Download do arquivo instalador do navegador Google Chrome e ao mesmo tempo renomeando-o como "InstaladorChrome.exe";

6. Exibição de tela referente ao diretório para onde o download foi executado (C:Itemporario); Após a compilação destes comandos basta apenas copiar o inject.bin criado para o cartão MicroSD que será inserido dentro do slot do Rubber Ducky. No momento da sua conexão a alguma máquina o disparo destes comandos será realizado de forma automática. O resultado da execução após a sua conexão pode ser observado na Figura 8. 
Figura 8 - Etapas de execução do Rubber Ducky após a sua conexão.

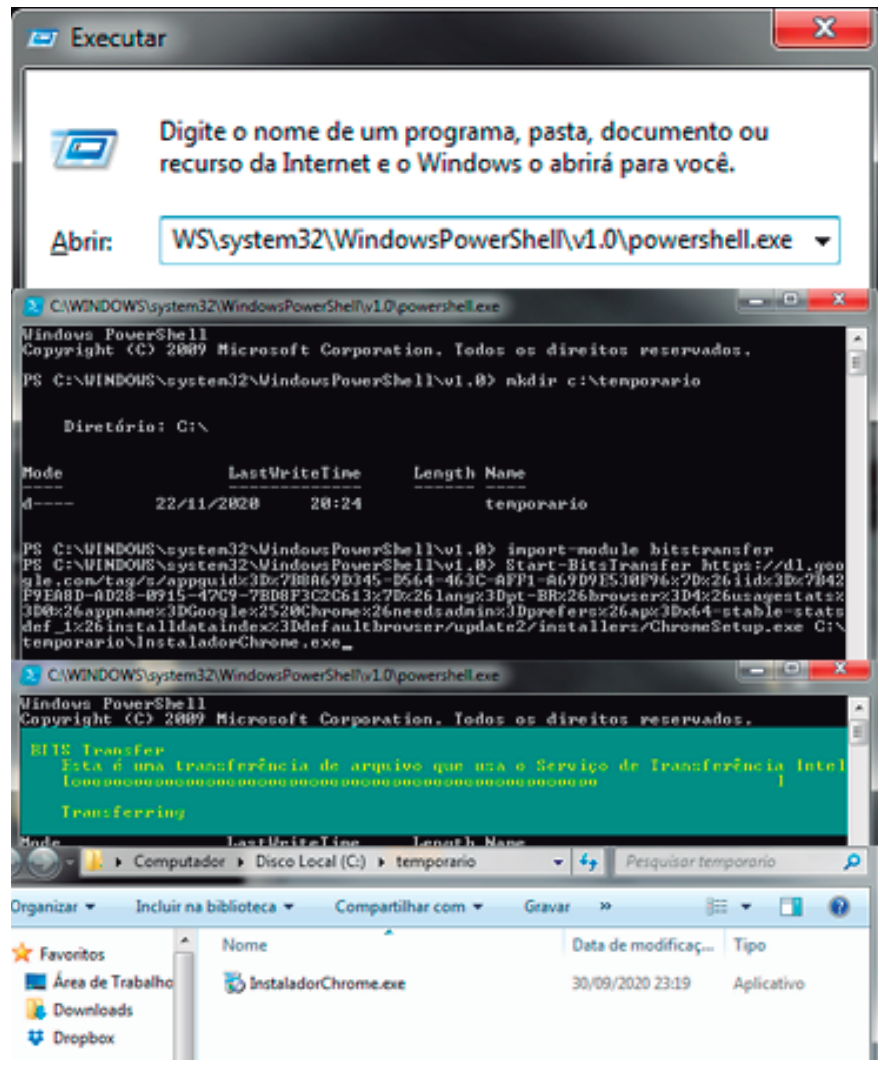

Fonte: Construção do autor.

Observando as etapas de execução do Rubber Ducky na Figura 8, pode-se detalhar os seguintes passos: invocação do PowerShell via caixa de diálogo Executar, criação do diretório "temporário" para onde o arquivo será baixado; criação de serviço para download do instalador; exibição do diretório para onde o arquivo foi baixado.

Figura 9 - Interrupção de execução do script do Rubber Ducky.

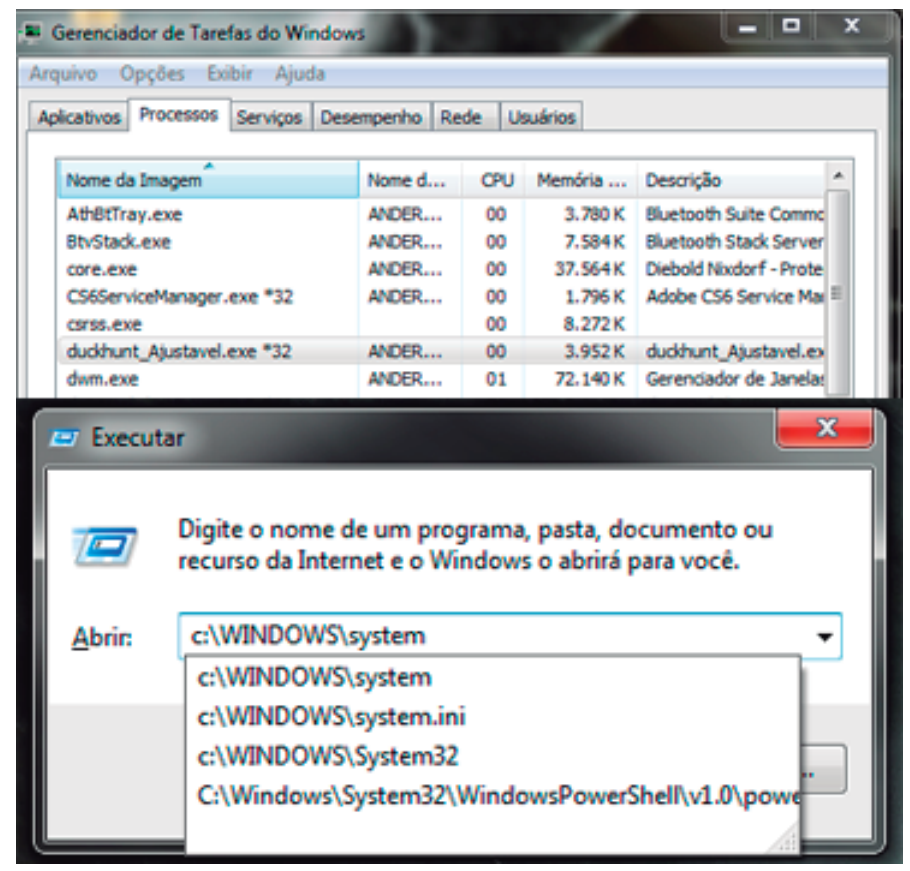

Fonte: Construção do autor. 
Finalmente depois da execução da solução desenvolvida para identificação do Rubber Ducky, a qual pode ser observada na Figura 9, tem-se o "Gerenciador de Tarefas" com execução do bloqueio e após a inserção do tamanho da palavra definida no arquivo "duckhunt.conf”, o sistema identificou que estava sendo vítima de um Rubber Ducky. Deste modo a execução do script foi interrompido e o restante das instruções não foram executadas.

\section{CONCLUSÃO}

Ao abordar o funcionamento de um dispositivo Rubber Ducky, pôde-se constatar que o mesmo desempenha com eficiência a execução das tarefas programadas através do Duck Toolkit.

Um cenário composto por uma sala equipada com vários computadores instalados e com informações privilegiadas armazenadas caracteriza-se como um potencial risco iminente para pessoas mal-intencionadas na obtenção de informações alheias. Basta um momento de descuido e conforme a simulação dos testes, a ação deste dispositivo pode ser instantânea. Com estes testes, expôs-se uma forma de penetração computacional extremamente eficiente, e ao mesmo tempo foi desenvolvida uma solução para tal bloqueio, completando a premissa Ethical Hacker, a qual consiste exatamente na identificação/solução de vulnerabilidades em ambientes de tecnologia da informação.

Por sua vez a versatilidade da linguagem Python, juntamente com seus módulos adicionais, como o PyHook e o Py2exe ofereceram uma solução opcional para impedir o sucesso da ação deste tipo de dispositivo por meio da interrupção de script. Assim, por meio de uma linguagem de programação chegou-se a uma solução parcial, não o interrompendo em seu funcionamento físico.

A segurança cibernética deve estar em constante revisão, comprovado pela exposição de mais um tipo de dispositivo, capaz de causar sérios danos na confidencialidade de informações.

\section{REFERÊNCIAS}

CANNOLS, B.;GHAFARIAN, A. Hacking Experiment by Using USB Rubber Ducky Scripting, Artigo, Revista Systemics, Cybernetics and Informatics, v. 15, n. 2, 2017. Disponível em: https://bit.ly/ 3e5kCE9. Acesso em: maio, 2020.

HAK5. USB Rubber Ducky. Disponível em: https://bit.ly/3ua7mUm. Acesso em: maio, 2020.

HARIANTO, H. E. e GUNAWAN, D. Wi-Fi password stealing program using USB rubber ducky, Artigo, TELKOMNIKA (Telecommunication, Computing, Electronics and Control), ISSN 1693-6930, v. 17, n. 2, Abril, 2019, p.745-752, Disponível em: https://bit.ly/2R88oSl. Acesso em: outubro, 2020. 
KONZEN, M. P. Gestão de riscos de Segurança da Informação baseada na Norma NBR ISSO/

IEC 27005 usando padrões de segurança, Dissertação de Mestrado em Engenharia de Produção, Universidade Federal de Santa Maria, 2013. Disponível em: https://bit.ly/3eHp4s5. Acesso em: abril, 2020.

MARCIANO, J. L. P. Segurança da Informação - uma abordagem social, Tese de doutorado em Ciência da Informação, Universidade de Brasília, 2006. Disponível em: https://bit.ly/3t8tXiM. Acesso em: abril, 2020.

OLIVEIRA, J. C. de. Ransomware - Laboratório de Ataque do WannaCry, Trabalho de Conclusão de Curso em Engenharia de Software, Universidade de Brasília, 2018. Disponível em: https:/bit.ly/ 3h57xgn. Acesso em: abril, 2020.

PAVAN, P. V. A. e GUARDIA, H. C. Pentest para auditoria de segurança de rede em ambientes corporativos, Artigo, Revista Tecnologias, Infraestrutura e Software, v. 4, n. 2, maio-ago, 2015. Disponível em: https://bit.ly/3t1vE1G. Acesso em: maio 2020.

PYHOOK. Página Inicial. Disponível em: https://bit.ly/3eNqWzx. Acesso em: out. 2020.

PYTHON: PY2EXE. Página Inicial. Disponível em: https://bit.ly/3u9SXaO. Acesso em: out. 2020.

ROCHA, A. T. Q. et al. Pentest para Quebra de Criptografia Wireless, Artigo, Caderno de Estudos Tecnológicos, n. 1, v. 6, ed. Junho, 2019. Disponível em: https://bit.ly/3xE5AwT. Acesso em: maio 2020.

SANTOS, R. E. C. dos. Laboratório Virtual Para Pentest Na Análise De Vulnerabilidade, Trabalho de Conclusão de Curso em Engenharia da Computação, Centro Universitário de Brasília, 2018. Disponível em: https://bit.ly/3udcuHu. Acesso em: maio 2020.

SILVA, C. A. da. Gestão da Segurança da Informação: um olhar a partir da Ciência da Informação, Dissertação, Mestrado em Ciência da informação, Pontifícia Universidade Católica, 2009. Disponível em: https://bit.ly/3ukJo9n. Acesso em: abr. 2020.

SILVA, R. O. da; SILVA, I. R. S. Linguagem de Programação Python, Artigo, Revista Tecnologias em Projeção, ISSN: 2178-6267, v. 10, n. 1, p. 55-71, 2019. Disponível em: https://bit.ly/3351Pp0. Acesso em: out. 2020. 
